\title{
A Comparative Study on Pragmatic Rules for Chinese and English Social Appellation
}

\author{
Yong Fang ${ }^{1,2}$ \\ 1. School of Humanity, Central China Normal University, \\ Wuhan, P.R. China, Postcode: 430079 \\ 875540144@qq.com \\ 2. Wuhan Language and Culture Research Center, Jianghan University, \\ Wuhan, P.R. China, Postcode: 430056
}

\begin{abstract}
Keywords: Social Appellation; Pragmatic Rules; Comparison between Chinese and English Languages; Power and Solidarity
\end{abstract}

\begin{abstract}
Based on the concepts of Chinese culture, e.g. 'if names not being rectified, what is said will sound unreasonable' and 'emphasizing on status and clarifying human relations', Chinese social appellation is affected by pragmatic factors including but not limited to gender, occupation, age, identity, occasion, motivation and emotion. English social appellation, based on its value of 'people oriented and names used', on the other hand, follows easier rules, and is more casual in use. The pragmatics of social appellation in two languages is subject to the principle of power and solidarity.
\end{abstract}

\section{Introduction}

Social relation, social roles and level of respect among the communication parties, as reflected by social appellation, are not interchangeable in linguistic performances. The choosing of appellation is restricted by language itself, its culture background, social psychology, etc., which is the result of interactions between linguistic performances and social relations. The differences in distance, closeness, hierarchy and age exist universally among individuals in any social group. The parties in social communication must obey the specific social rules and choose the most appropriate social appellation under the guidance of such rules, so as to clarify their social status and to distinguish interpersonal relations, based on which, smooth social communicative activities are carried out.

American social psychologists R. Brown and A. Gilman (1960) were the first to point out that the selection of social appellation is restricted by national identities, social structures and group consciousness, and is also relevant to the gap of social status and degree of closeness among communicators [1]. R. Brown and A. Gilman refined interpersonal relationship in the social intercourse into power (unequal relation) and solidarity (equal relation). If $\mathrm{A}$ is able to control the behavior of $\mathrm{B}$, then $\mathrm{A}$ possesses power over $\mathrm{B}$ and their relationship is defined as power. The society we live in is full of various kinds of hierarchies. Cultural tradition, ideology, religious beliefs, social status, age, gender, race, occupation and even the amount of professional knowledge in specific fields can be the source of power, in its abstract sense. Under such concepts of power, in addition to the referential meaning of its cognitive concept, social appellation also needs to be in accordance with the identity function within its sociology concept [2]. Solidarity, on the other hand, refers to an equal relation generated by common grounds of $\mathrm{A}$ and $\mathrm{B}$ in some respects. This essay aims at the comparison of pragmatic rules for social appellation in Chinese and English language, the result of which will play a referential role on cross-cultural communication of Chinese and English, bilingual translation of social appellation between Chinese and English, TCFL of Chinese social appellation and teaching of English appellation in China.

\section{Pragmatic Rules of Social Appellation in two Languages}

Pragmatic Rules for Chinese Social Appellation. Based on and maintained by blood relations, Chinese culture is group oriented, emphasizing interpersonal relationships among its members. 
Compared with its kinship terms, the pragmatic rules of Chinese social appellation are far more complicated, which, in the setting of social intercourse, is a reflection of China's social conception of respect for seniority, and intimacy of people being different [3]. Chinese social appellation is comprehensively affected by gender, occupation, age, status, occasion, motivation and emotion of communicators [4]. These factors function not only individually, but simultaneously as well, thus causing the changes in the usage of appellation. Yet, generally speaking, the pragmatic rules of Chinese social appellation fundamentally obey the principle of power and solidarity. The use of social appellation in Chinese abides by the following pragmatic rules:

Normally, respectful appellations are adopted for seniors and superiors, that is people who have power over the speaker. Polite and respectful appellations are adopted for strangers and alienate people [5]. One adopts normal appellations or appellations which shows intimacy for juniors, subordinates and people of the speaker's same status. For instance, one calls his/her boss job title+surname (e.g. Director Zhang). As for the colleagues younger than oneself, one calls him/her Xiao+surname (e.g. Xiao Zhang), or full name (e.g. Zhang Ming). In a hospital, one calls the medical staff job title+surname (e.g. Doctor Wang, Nurse Li) or Comrade+job title (e.g. Comrade Nurse).

Formal and respectful appellations are used in formal occasions, while intimate appellations are adopted in informal settings [6]. For instance, in formal occasions, one calls a familiar university faculty Professor+surname (e.g. Professor Li), or Doctor+surname (e.g. Doctor Li). In informal settings, those appellations will be replaced by given name (e.g. Hongjian), given name+elder brother (Xiong) (e.g. Hongjian Xiong), surname+Elder Brother (Xiong) (e.g. Fang Xiong).

The longer the appellation, the more formal, respectful and serious it is, and at the same time, the more distant the relation sounds to be. Conversely, the shorter the appellation, the more casual and intimate the expression is. For example, Sun Roujia Lao Shi (fullname+job title), Sun Lao Shi (surname+job title) and Roujia (given name) distinctively express the change of speaker's emotion and his/her intimacy with the listener. The decrease in syllables embodies a more intimate and casual relationship in social intercourse [7].

If a person has a job title, academic title and degree title at the same time, Chinese call him/her by his/her job title. For example, if a university President is a professor and a $\mathrm{Ph} . \mathrm{D}$ at the same time, we normally call him/her job surname+title (e.g. Wang Xiao Zhang).

When the element of age conflicts with position, the influence of position takes the priority. For instance, Wang Xiaomao is younger than Zhang Dajin, but Wang's job position is higher than Zhang's. Under such circumstance, since Wang Xiaomao possesses power over Zhang Dajin, he then uses intimate and normal appellation, such as Lao Zhang (Lao+surname) to address Zhang Dajin. Zhang Dajin, on the other hand, should use respectful appellation, such as Wang Chu Zhang (surname+job title) to address Wang Xiaomao instead.

When the addressee is in a deputy or an associate position, the word deputy, associate or vice does not normally appear in the social appellation. For example, Deputy Director Li is called as Li Chu Zhang (surname+job title without Deputy), and Associate Professor Wang will be called as Wang Jiao Shou (Professor Wang, surname+job title without Associate)

Among social appellations in job title, academic title and degree title, some of them are used face to face, but others cannot be used that way. The principle of reserving the superior title and abandoning the inferior title is applied in face to face communication. For instance, Professor, an academic title, is used face to face. But the use of academic titles such as Lecturer or Teaching Assistant with a faculty's name is inappropriate in the university. As for degree title, Ph.D degree is used face to face, but Master's and Bachelor's degree are not. In hospitals, the academic title Chief Physician is used either with one's full name or surname, e.g. Wang Zhu Ren (Chief Physicia Wang), Wang Tianyi Zhu Ren (Chief Physician Wang Tianyi). Inferior academic titles such as Physician, however, is not used with a person's name in the face to face communication with the person.

Pragmatic Rules for English Social Appellation. Social appellation in English is a reflection of cultures of English speaking countries in its social intercourse. Cultures of English speaking 
countries admire individuality, and its concept of equality boasts a long history and is deeply rooted in the heart of its people. The Declaration of Independence of America, which was written in 1776, proposes that 'all men are created equal'. The Anglo-American social relationship is based on contractual relation, focusing on individualism and emphasizing equality among its citizens. The social appellations in English language reflect exactly the beliefs, concepts and culture of its people [8]. In Chinese culture, people believe that if names are not rectified, what is said will not sound reasonable, and that emphasizing on status and clarifying human relations. Social appellations in English are based on the value of its culture too, which is quoted as 'people oriented, names used'. The use of English social appellation is more casual and simple than that of Chinese social appellation. The points below summarize the pragmatic rules obeyed by social appellation in English language:

People address one another directly with name in ordinary occasions, which embodies the equality, causality and intimacy in its interpersonal relations. For example, a university professor whose name is Mike Smith is addressed by his colleagues, friends and even students as Mike (given name) in ordinary occasions.

In formal occasions, the following two models of social appellation are used. (a) job title/academic title/military rank + family name/full name. (b) Mr./Miss/Mrs./Ms.+ family name/full name. When job titles can be used as an appellation, model (a) applies. Model (b) works when job titles cannot be used as appellation.

Model (a) and (b), as in, are also used to show respect to the addressee, emphasizing his/her higher position and power over the speaker himself/herself. For instance, in order to show respect to Mike Smith, a university professor, his students address him Professor Smith (job title + surname).

Respectful appellations are used for strangers, e.g. sir, madam, lady, etc.

\section{Comparison of Pragmatic Rules for Chinese and English Social Appellation}

Even though social appellations in Chinese and English share some similarities in their pragmatic use, their differences are apparent as well. It is not common for Chinese to address people by their names, whether family name or full name, directly. The use of full name and family name exists in circumstances below. Firstly, they are used between friends and relatives, showing intimacy between the speakers. Secondly, they are used by the elder generation towards the younger generation, or by a person in a senior position on his/her subordinates. The surface structure of Chinese names also has impact on the use if itself. A given name is used to address a person only when it is of double Chinese characters. If the given name is of only one Chinese character, it must be used together with the person's surname. For instance, a man named Zhu Jun (surname +given name) is addressed by his full name, Zhu Jun, other than Jun(given name). A man named Wang Zhixun (given name is of two Chinese characters, Zhi and Xun) can be addressed either by his given name, Zhixun, or his full name, Wang Zhixun.

In English social appellation, however, the use of given name or full name is much more frequent. They are used to address almost all you social relations, including the elder generations, your boss in the company, or someone you just knew. For instance, when a college student addresses his professor by his/her given name, the professor will not feel angry at all. Instead, the professor feels that the image he/she leaves on his/her students is that of kind, intimate and easygoing.

When we move to appellations used in the workplace, we find that there are around 50 job titles or professional titles in Chinese language. Most of these 50 titles can be used as appellation and the use of them is wide across different settings of social intercourse. Since China is an official rank and power oriented society, so people pay more attention to power relations. It is no wonder that job titles like President+surname, Director+surname etc. can be commonly heard in all kinds of social intercourse [9]. The extrusive use of the official position in occupational appellation is much affected and restricted by such social cultural mentality.

It is a different case in culture of English speaking countries. As mentioned above, the culture of English countries emphasizes individual ideology and advocates that all men are created equal, that everyone is equal, and that everyone is free. Culture in English speaking countries, comparatively, 
pays more attention to equality and to narrow the social difference. Thus, the social differences between students and their teachers, employees and employers are deemed an obstacle for the cultural believe. The number of occupations and job titles used for appellation are therefore very limited in English language. The few examples include President, Professor and Doctor. Appellations used to make it up are the frequent use of Mr./Ms./Mrs./Miss+ full name/family name. These structures are still often used, even though the addressee can be addressed by his/her job title+surname / full name.

The use of Chinese fictive kinship appellation is different from that in English. Addressing non-kinship or even strangers by kinship terms is common, and has been a part of China's tradition . $\mathrm{Hu}$ Hongli and Jin Jing'ai collect some widely used fictive kinship terms, which includes Ye Ye (grandpa), Nai Nai (grandma), Bo Bo (elder uncle), Shu Shu (younger uncle), A Yi (aunt), Da Ge (elder brother), Da Sao (elder brother's wife), Da Jie (elder sister), Xiao Mei (younger sister) [10]. The choice of fictive kinship terms is based on the gender, age, social character of the addressee, and the degree of familiarity between the speakers. From the Chinese feudal society until now, kinship relation has been one of the important kinds of relations and power used to maintain social stability. Chinese people hope that kinship relation can serve as a bound to connect all the social members and build a community like a bigger family. Kinship terms are thus applied to all walks of life, which leads to the generalization of them.

Individualism is much admired in English speaking countries. People emphasize private interest and respect the ideology of free competition. Compared with China, the connection within family members in English speaking countries is weak, and interpersonal social relations are not as close as that in China's tradition. People do not have the intention to treat other members in the society as their relatives. As a result, the use of fictive kinship terms on people who are not one's relatives is considered inappropriate under most circumstances, and therefore rarely seen in social intercourse. Wang Meiqiong points out that when children address their friends' parents, Mr./Mrs +family name are used. Only when the seniors of both families are very close, do the children address the parents of the other family by the structure of Uncle (Aunt) + family name. However, the adults in English speaking countries never address non-relatives by fictive kinship terms, and use the appellation structure Mr./Mrs +family name instead.

\section{Conclusion}

This paper compares the pragmatic rules of Chinese and English social appellations. The use of names for social appellation is much more frequent in English language than in Chinese. The use of occupational and job titles in Chinese is much more frequent than that in English, reflecting Chinese people's focus on official rank and power. Addressing non-kinship or even strangers by kinship terms is common, and has been a part of China's tradition. The use of fictive kinship terms on people who are not one's relatives is considered inappropriate under most circumstances in English. The use of social appellations in both Chinese and English language is subject to power and solidarity.

\section{Reference}

[1] R. Brown and A. Gilman: The Pronouns of Power and Solidarity, in T.A. Sebeok (ed.), Style in Language, MIT Press, pp. 253-76, 1960

[2] Qingyuan. Li and Xiaohui. Li: Comparison of Difference among Appellation Pragmatic Use in the Perspective of Cross Culture in English and Chinese, Journal of Chongqing Normal University (Philosophy and Social Science Edition), 2008(3) pp.110-114

[3] Lishan. Liu: Comparison of Pragmatic Function in the Appellation of Chinese and English under Social Context, Journal of Yanshan University (Philosophy and Social Science Edition), Vol.7 (2006) No.1, pp.32-34 
[4] Haitang. Ren, Ningxia. Feng and Ronghua. Wang: Comparison and Analysis of Social Appellation in Chinese and English. Journal of Northwest University (Philosophy and Social Science Edition) Vol. 34 (2004) No.1, pp.172-175

[5] Junxia. Wang: A Study of the Relationship between Language and Social Element from the Perspective of Appellation and Its Changes in Chinese and English, Journal of Qiqihaer University (Philosophy and Social Science Edition), 2004(1) pp.110-111

[6] Meiqiong. Wang: The Pragmatic Comparison and Translation of Social Appellation in English and Chinese, Journal of Huangshi Institute of Technology (Humanity and Social Science Edition), 2008(1) pp.69-73

[7] Huigang. Tian: Chinese and Western Interpersonal Appellation System, Beijing: Foreign Language Teaching and Research Press, 1998

[8] Hongli. Hu: The Application of Social Appellation in Cross Cultural Communication, The Journal of Xinjiang Normal University (Philosophy and Social Science Edition), Vol 24 (2003) pp.102-105

[9] Meiqiong. Wang: A Study on the Differences between English and Chinese Social Appellation, Journal of Huangshi Institute of Technology (Humanity and Social Science Edition), Vol. 24 (2007) pp.100-103

[10] Jing'ai. Jin: Analysis of Pragmatic Comparison about Appellation in Chinese and English, Journal of Xiangtan Normal College (Social Science Edition), Vol. 30 (2008) pp.227-229. 